\title{
CONVERGENCE OF DIFFERENCE APPROXIMATIONS AND NONLINEAR SEMIGROUPS
}

\author{
SHIGEO KANDA \\ (Communicated by Palle E. T. Jorgensen)
}

\begin{abstract}
We give a convergence theorem for the difference approximation for the evolution equation $(d / d t) u(t) \in A u(t)$ and a generation theorem of nonlinear semigroups for directed dissipative operators $A$ in a real Hilbert space.
\end{abstract}

\section{INTRODUCTION}

In this paper we consider the evolution equation:

$$
(d / d t) u(t) \in A u(t), \quad t \in[0, T),
$$

for a given (multivalued) nonlinear operator $A$ in a real Hilbert space.

After a pioneering work of Kōmura [4], many authors have treated the generation of nonlinear semigroups having dissipative or $\omega$-dissipative operator as generators. Our main purpose is to introduce a notion of directed $L$-dissipative operators as a generalization of $\omega$-dissipative operators and to give a convergence theorem for difference approximations for (DE) and a generation theorem of nonlinear semigroups through it. In $\S 1$ we state definitions and a convergence theorem for difference approximation for $(\mathrm{DE})$ and the proofs are given in $\S 2$. In $\S 3$ we give a generation theorem of nonlinear semigroups through the convergence theorem in $\S 1$.

\section{Definitions AND CONVERgENCE OF DIFFERENCE APPROXIMATIONS}

Let $H$ be a real Hilbert space with inner product $\langle\cdot, \cdot\rangle$ and norm $\|\cdot\|$. Let $A$ be a (multivalued) operator in $H$ with domain $D(A)$ and range $R(A)$. We say that $A$ is directed $L$-dissipative if the following conditions are satisfied:

(i) for every $\lambda>0,(1-\lambda A)^{-1}$ is a single-valued operator.

(ii) $\left\langle x^{\prime}-y^{\prime}, x-y\right\rangle \leq L(\|x-y\|)\|x-y\|$ for $x, y \in D(A), x^{\prime} \in A x$, and $y^{\prime} \in A y$, where $L$ is an increasing function such that $L(0)$ equals zero and satisfies the condition, $\int_{0}^{1} d t / L(t)=\infty$.

Received by the editors January 26, 1989.

1980 Mathematics Subject Classification (1985 Revision). Primary 47H10; Secondary 34A34.

Key words and phrases. Dissipative, evolution equation, semigroup, Hilbert space. 
(iii) $\left\langle x^{\prime}-x^{\prime \prime}, \lambda x^{\prime}\right\rangle \leq 0$ for $\lambda>0, x \in D(A), x^{\prime} \in A x, x-\lambda x^{\prime} \in D(A)$, and $x^{\prime \prime} \in A\left(x-\lambda x^{\prime}\right)$.

We note that condition (iii) is an analogue to that of time-like analyticity in Furuya [2]. Our main theorem is the following:

Theorem 1. Let $A$ be a directed $L$-dissipative operator in $H$ satisfying the range condition

$$
R(1-\lambda A) \supset D(A) \text { for all } \lambda>0 .
$$

Let $T>0$ and $T=n h_{n}$, where $n$ is a positive integer. Let $u_{0} \in D(A)$. We define the simple functions $u_{n}$ on $[0, T]$ by

$$
u_{n}(t)=\left(1-h_{n} A\right)^{-k} u_{0} \text { for } t \in\left((k-1) h_{n}, k h_{n}\right] \text { and } u_{n}(0)=u_{0} \text {. }
$$

Then $\left\{u_{n}(t)\right\}$ converges uniformly on $[0, T]$ as $n \rightarrow \infty$ and if we let $u$ be the limit function of $\left\{u_{n}\right\}$, then $u$ is a Lipschitz continuous function with Lipschitz constant $\left|A u_{0}\right| \equiv \inf \left\{\|z\| \mid z \in A u_{0}\right\}$, that is,

$$
\|u(t)-u(s)\| \leq\left|A u_{0}\right||t-s| \text { for } t, s \in[0, T] .
$$

\section{Proof of TheOREM 1}

We begin with the following lemma.

Lemma 1.1. Let $A$ be a directed L-dissipative operator in $H$ satisfying the range condition ( $\mathrm{R} 1)$. Let $x_{0}, y_{0} \in D(A)$. Let $h, k$ be positive numbers such that $h=l k$, where $l$ is a positive integer. Put $x_{n+1}=(1-h A)^{-1} x_{n}$ and $y_{m+1}=$ $(1-k A)^{-1} y_{m}$ for $n, m=0,1,2, \ldots$ Let $M=\max \left(\left|A x_{0}\right|+\left|A y_{0}\right|,\left|A y_{0}\right|^{2}\right)$, where $|A x| \equiv \inf \left\{\left\|x^{\prime}\right\| \mid x^{\prime} \in A x\right\}$. Let $\varepsilon$ be a given positive number. If $\left\|x_{n}-y_{l n}\right\| \geq \varepsilon$ and $M h<\min (\varepsilon / 2, L(\varepsilon) \varepsilon)$, then the following estimate holds:

$$
\left\|x_{n+1}-y_{l(n+1)}\right\|-\left\|x_{n}-y_{l n}\right\| \leq 3 h L\left(\left\|x_{n}-y_{l n}\right\|+M h\right) \text {. }
$$

Proof. Let $x_{n+1}^{\prime} \in A x_{n+1}$. Let $p$ be a nonnegative integer. Choose $y_{l(n+1)-p}^{\prime} \in$ $A y_{l(n+1)-p}$ such that

$$
y_{l(n+1)-p}=y_{l(n+1)-p-1}+k y_{l(n+1)-p}^{\prime} .
$$

Then

$$
\begin{aligned}
\left\|x_{n+1}-p k x_{n+1}^{\prime}-y_{l(n+1)-p}\right\|^{2}= & \| x_{n+1}-(p+1) k x_{n+1}^{\prime}-y_{l(n+1)-p-1} \\
& +k\left(x_{n+1}^{\prime}-y_{l(n+1)-p}^{\prime}\right) \|^{2} \\
= & \left\|x_{n+1}-(p+1) k x_{n+1}^{\prime}-y_{l(n+1)-p-1}\right\|^{2} \\
& +2 k\left\langle x_{n+1}^{\prime}-y_{l(n+1)-p}^{\prime}, x_{n+1}-y_{l(n+1)-p}\right\rangle \\
& -(p+1) k^{2}\left\|x_{n+1}^{\prime}-y_{l(n+1)-p}^{\prime}\right\|^{2}-p k^{2}\left\|x_{n+1}^{\prime}\right\|^{2} \\
& +p k^{2}\left\|y_{l(n+1)-p}^{\prime}\right\|^{2} \text { for } p=0,1,2, \ldots, l-1 .
\end{aligned}
$$


Choose $x_{n+1}^{\prime} \in A x_{n+1}$ such that $x_{n}=x_{n+1}-h x_{n+1}^{\prime}$. Then, for any $x_{n}^{\prime} \in A x_{n}=$ $A\left(x_{n+1}-h x_{n+1}^{\prime}\right)$,

$$
0 \geq\left\langle x_{n+1}^{\prime}-x_{n}^{\prime}, x_{n+1}^{\prime}\right\rangle=\left\|x_{n+1}^{\prime}\right\|^{2}-\left\langle x_{n}^{\prime}, x_{n+1}^{\prime}\right\rangle \geq\left\|x_{n+1}^{\prime}\right\|^{2}-\left\|x_{n}^{\prime}\right\|\left\|x_{n+1}^{\prime}\right\| .
$$

Thus the following estimates hold:

$$
\begin{aligned}
\left\|x_{n+1}^{\prime}\right\| & \leq\left\|x_{n}^{\prime}\right\| \leq\left|A x_{0}\right| ; \\
\left\|x_{n+1}-x_{n}\right\| & =\left\|h x_{n+1}^{\prime}\right\| \leq h\left|A x_{0}\right| ; \\
\left|\left\|x_{n+1}-y_{l(n+1)-p}\right\|-\left\|x_{n}-y_{l n}\right\|\right| & \leq\left\|x_{n+1}-x_{n}\right\|+\sum_{j=1}^{l-p}\left\|y_{l n+j}-y_{l n+j-1}\right\| \\
& \leq h\left|A x_{0}\right|+(l-p) k\left|A y_{0}\right| \\
& \leq M h \text { for } p=0,1,2, \ldots, l .
\end{aligned}
$$

From (3), using (4), (5), (6), and (ii) we have

$$
\begin{aligned}
& \left\|x_{n+1}-p k x_{n+1}^{\prime}-y_{l(n+1)-p}\right\|^{2}-\left\|x_{n+1}-(p+1) k x_{n+1}^{\prime}-y_{l(n+1)-p-1}\right\|^{2} \\
& \quad \leq 2 k L\left(\left\|x_{n}-y_{l n}\right\|+M h\right)\left(\left\|x_{n}-y_{l n}\right\|+M h\right)+M p k^{2} .
\end{aligned}
$$

Adding these inequalities for $p=0,1,2, \ldots, l-1$, we get

$$
\begin{aligned}
& \left\|x_{n+1}-y_{l(n+1)}\right\|^{2}-\left\|x_{n}-y_{l n}\right\|^{2} \\
& \quad \leq 2 h L\left(\left\|x_{n}-y_{l n}\right\|+M h\right)\left(\left\|x_{n}-y_{l n}\right\|+M h\right)+M l(l-1) k^{2} / 2 \\
& \quad \leq 2 h L\left(\left\|x_{n}-y_{l n}\right\|+M h\right)\left(\left\|x_{n}-y_{l n}\right\|+M h\right)+M h^{2} .
\end{aligned}
$$

In addition, if $\left\|x_{n}-y_{l n}\right\| \geq \varepsilon>2 M h$, then using (6) we have

$$
\begin{aligned}
& \left\|x_{n+1}-y_{l(n+1)}\right\|^{2}-\left\|x_{n}-y_{l n}\right\|^{2} \\
& \quad=\left(\left\|x_{n+1}-y_{l(n+1)}\right\|-\left\|x_{n}-y_{l n}\right\|\right)\left(\left\|x_{n+1}-y_{l(n+1)}\right\|+\left\|x_{n}-y_{l n}\right\|\right) \\
& \quad \geq\left(\left\|x_{n+1}-y_{l(n+1)}\right\|-\left\|x_{n}-y_{l n}\right\|\right)\left(2\left\|x_{n}-y_{l n}\right\|-M h\right) \\
& \quad \geq\left(\left\|x_{n+1}-y_{l(n+1)}\right\|-\left\|x_{n}-y_{l n}\right\|\right)\left(\left\|x_{n}-y_{l n}\right\|+M h\right) .
\end{aligned}
$$

Thus, if $\left\|x_{n}-y_{l n}\right\| \geq \varepsilon>2 M h$ and $M h<L(\varepsilon) \varepsilon$, then

$$
\begin{aligned}
\left\|x_{n+1}-y_{l(n+1)}\right\|-\left\|x_{n}-y_{l n}\right\| & \leq 2 h L\left(\left\|x_{n}-y_{l n}\right\|+M h\right)+M h^{2} / \varepsilon \\
& \leq 2 h L\left(\left\|x_{n}-y_{l n}\right\|+M h\right)+h L(\varepsilon) \\
& \leq 3 h L\left(\left\|x_{n}-y_{l n}\right\|+M h\right) .
\end{aligned}
$$

Lemma 1.2. Let $A, x_{0}$, and $y_{0}$ be as in Lemma 1.1. Let $T>0$ and $T=n h_{n}$, where $n$ is a positive integer. Then for any $\varepsilon>0$ there exist a positive number $\delta=\delta(\varepsilon)$ and a positive integer $N=N\left(\varepsilon, x_{0}, y_{0}\right)$ such that

$$
\left\|\left(1-\frac{t}{n} A\right)^{-n} x_{0}-\left(1-\frac{t}{\ln } A\right)^{-l n} y_{0}\right\| \leq \varepsilon
$$

for $\left\|x_{0}-y_{0}\right\| \leq \delta, n \geq N, l=1,2,3, \ldots$, and $t \in[0, T]$. 
Proof. Given $\varepsilon>0$, choose $\eta=\eta(\varepsilon)>0$ such that $\int_{\eta}^{\varepsilon} d y / L(y) \geq 4 T$. Setting $\delta=\delta(\varepsilon)=\eta / 3$, choose $x_{0}, y_{0} \in D(A)$ such that $\left\|x_{0}-y_{0}\right\| \leq \delta$. Moreover, choose a positive integer $N=N\left(\varepsilon, x_{0}, y_{0}\right)$ such that $M h_{N}=M T / N<$ $\min (\eta / 2, L(\varepsilon) \varepsilon)$. For simplicity, put

$$
\alpha_{k}^{n}(t)=\left\|\left(1-\frac{t}{n} A\right)^{-k} x_{0}-\left(1-\frac{t}{\ln } A\right)^{-l k} y_{0}\right\| \text { for } t \in[0, T] .
$$

Let $n \geq N$ and assume that $\alpha_{n}^{n}(t)>\varepsilon$ for some $t \in[0, T]$. Because $\alpha_{0}^{n}(t)=$ $\left\|x_{0}-y_{0}\right\| \leq \eta / 3$ and by noting (6) we have

$$
\begin{aligned}
\alpha_{n-1}^{n}(t) & =\alpha_{n}^{n}(t)+\alpha_{n-1}^{n}(t)-\alpha_{n}^{n}(t) \\
& \geq \varepsilon-M t / n \geq \varepsilon-M h_{N} \\
& >\eta-\eta / 2=\eta / 2,
\end{aligned}
$$

there exists a nonnegative integer $k_{0}<k \leq n$ such that

$$
\alpha_{k_{0}}^{n}(t)<\eta / 2 \text { and } \alpha_{k}^{n}(t) \geq \eta / 2 \text { for } k_{0}<k \leq n .
$$

For $t \in(0, T]$ and putting $h=t / n$ we have

$$
\begin{aligned}
4 T & \leq \int_{\eta}^{\varepsilon} \frac{d y}{L(y)} \leq \int_{\alpha_{k_{0}}^{n}(t)+M h}^{\alpha_{n}^{n}(t)+M h} \frac{d y}{L(y)} \\
& \leq \sum_{k=k_{0}}^{n-1} \frac{\alpha_{k+1}^{n}(t)+M h-\left(\alpha_{k}^{n}(t)+M h\right)}{L\left(\alpha_{k}^{n}(t)+M h\right)} \\
& \leq \sum_{k=k_{0}}^{n-1} 3 h=3\left(n-k_{0}\right) h \leq 3 t \leq 3 T, \quad \text { by Lemma } 1.1 .
\end{aligned}
$$

This is a contradiction. Hence $\alpha_{n}^{n}(t) \leq \varepsilon$ for $n \geq N$ and $t \in[0, T]$.

Proof of Theorem 1. Given $\varepsilon>0$, put $\delta(\varepsilon)=\varepsilon / 2\left(\left|A u_{0}\right|+1\right)$. Put $x_{k}^{n}=$ $\left(1-h_{n} A\right)^{-k} u_{0}$. Let $n, m \geq 2 T\left|A u_{0}\right| / \varepsilon \equiv N_{1}(\varepsilon)$. In the case $t \in[0, \delta(\varepsilon)]$, taking the integer $k_{n}$ so that $t \in\left(\left(k_{n}-1\right) h_{n}, k_{n} h_{n}\right]$, we obtain

$$
\begin{aligned}
\left\|u_{n}(t)-u_{0}\right\| & =\left\|x_{k_{n}}^{n}-u_{0}\right\|=\left\|\sum_{j=1}^{k_{n}}\left(x_{j}^{n}-x_{j-1}^{n}\right)\right\| \\
& \leq \sum_{j=1}^{k_{n}}\left\|x_{j}^{n}-x_{j-1}^{n}\right\| \leq \sum_{j=1}^{k_{n}} h_{n}\left|A u_{0}\right| \\
& \leq k_{n} h_{n}\left|A u_{0}\right| \leq\left(t+h_{n}\right)\left|A u_{0}\right| \\
& \leq \varepsilon / 2+\varepsilon / 2=\varepsilon .
\end{aligned}
$$

Similarly we have

$$
\left\|u_{m}(t)-u_{0}\right\| \leq \varepsilon
$$


Thus

$$
\begin{aligned}
\left\|u_{n}(t)-u_{m}(t)\right\| & \leq\left\|u_{n}(t)-u_{0}\right\|+\left\|u_{0}-u_{m}(t)\right\| \\
& \leq \varepsilon+\varepsilon=2 \varepsilon
\end{aligned}
$$

for $t \in[0, \delta(\varepsilon)]$ and $n, m \geq N_{1}(\varepsilon)$.

In the case $t \in[\delta(\varepsilon), T]$, by Lemma 1.2 there exists a positive integer $N_{2}(\varepsilon)$ such that

$$
\left\|\left(1-\frac{t}{k_{n}} A\right)^{-k_{n}} u_{0}-\left(1-\frac{t}{m k_{n}} A\right)^{-m k_{n}} u_{0}\right\| \leq \varepsilon \text { and } h_{n}\left|A u_{0}\right| \leq \varepsilon
$$

$$
\text { for } t \in[\delta(\varepsilon), T] \text { and } n, m \geq N_{2}(\varepsilon) \text {. }
$$

Thus

$$
\begin{aligned}
\left\|u_{n}(t)-u_{n m}(t)\right\|= & \left\|\left(1-h_{n} A\right)^{-k_{n}} u_{0}-\left(1-h_{n m} A\right)^{-k_{n m}} u_{0}\right\| \\
\leq & \left\|\left(1-\frac{k_{n} h_{n}}{k_{n}}(A)\right)^{-k_{n}} u_{0}-\left(1-\frac{k_{n} h_{n}}{m k_{n}} A\right)^{-m k_{n}} u_{0}\right\| \\
& +\left\|\left(1-h_{n m} A\right)^{-m k_{n}} u_{0}-\left(1-h_{n m} A\right)^{-k_{n m}} u_{0}\right\| \\
\leq & \varepsilon+\left\|\left(1-h_{n m} A\right)^{-m k_{n}} u_{0}-\left(1-h_{n m} A\right)^{-k_{n m}} u_{0}\right\| \\
= & \varepsilon+\left\|\sum_{j=k_{n m}+1}^{m k_{n}}\left(x_{j}^{n m}-x_{j-1}^{n m}\right)\right\| \\
& \leq \varepsilon+\sum_{j=k_{n m}+1}^{m k_{n}}\left\|x_{j}^{n m}-x_{j-1}^{n m}\right\| \\
& \leq \varepsilon+m h_{n m}\left|A u_{0}\right|=\varepsilon+h_{n}\left|A u_{0}\right| \leq 2 \varepsilon
\end{aligned}
$$

for $t \in[\delta(\varepsilon), T]$ and $n, m \geq N_{2}(\varepsilon)$. Similarly we have

$$
\left\|u_{n m}(t)-u_{m}(t)\right\| \leq 2 \varepsilon
$$

for $t \in[\delta(\varepsilon), T]$ and $n, m \geq N_{2}(\varepsilon)$.

Putting $N(\varepsilon)=\max \left(N_{1}(\varepsilon), N_{2}(\varepsilon)\right)$,

$$
\left\|u_{n}(t)-u_{m}(t)\right\| \leq 4 \varepsilon
$$

for $t \in[0, T]$ and $n, m \geq N(\varepsilon)$. Hence $\left\{u_{n}(t)\right\}$, converges uniformly on $[0, T]$. Put $u(t)=\lim _{n \rightarrow \infty} u_{n}(t)$ for $t \in[0, T]$. For $0<s<t \leq T$, choose integers $j, k$ such that $s \in\left((j-1) h_{n}, j h_{n}\right]$ and $t \in\left((k-1) h_{n}, k h_{n}\right]$. Then

$$
\begin{aligned}
\left\|u_{n}(t)-u_{n}(s)\right\| & =\left\|x_{k}^{n}-x_{j}^{n}\right\| \leq \sum_{i=j}^{k-1}\left\|x_{i+1}^{n}-x_{i}^{n}\right\| \leq \sum_{i=j}^{k-1} h_{n}\left|A u_{0}\right| \\
& \leq(k-j) h_{n}\left|A u_{0}\right| \leq\left(t-s+h_{n}\right)\left|A u_{0}\right| .
\end{aligned}
$$

Thus

$$
\|u(t)-u(s)\| \leq(t-s)\left|A u_{0}\right| \quad \text { as } n \rightarrow \infty
$$


This holds for $s=0$. Hence,

$$
\|u(t)-u(s)\| \leq\left|A u_{0}\right||t-s| \text { for all } t, s \in[0, T] .
$$

\section{GENERATION OF NONLINEAR SEMIGROUPS}

Let $A$ be a directed $L$-dissipative operator in $H$ satisfying the condition (R1). Following Benilan [1], we define an integral solution $u$ to the Cauchy problem:

$$
\left(C P_{T} ; u_{0}\right)\left\{\begin{array}{l}
(d / d t) u(t) \in A u(t) \quad \text { for } t \in[0, T), 0<T \leq \infty, \\
u(0)=u_{0}
\end{array}\right.
$$

as a continuous function on $[0, T)$ with $u(0)=u_{0}$ satisfying the inequality

$$
\begin{aligned}
\| u(t) & -x_{0}\left\|^{2}-\right\| u(s)-x_{0} \|^{2} \\
& \leq 2 \int_{s}^{t}\left\{\left\langle y_{0}, u(\tau)-x_{0}\right\rangle+L\left(\left\|u(\tau)-x_{0}\right\|\right)\left\|u(\tau)-x_{0}\right\|\right\} d \tau
\end{aligned}
$$

for $x_{0} \in D(A), y_{0} \in A x_{0}$, and $0 \leq s \leq t<T$.

It is easy to see that the limit function $u$ as in Theorem 1 is an integral solution to $\left(C P_{T} ; u_{0}\right)$, and satisfies

$$
\|v(t)-u(t)\|-\|v(s)-u(s)\| \leq \int_{s}^{t} L(\|v(\tau)-u(\tau)\|) d \tau,
$$

$0 \leq s \leq t<T$ for any integral solution $v(t)$ to $\left(C P_{T} ; v_{0}\right)$. Such a solution is called a mild solution of $\left(C P_{T} ; u_{0}\right)$.

Lemma 3.1. Let $u(t)=u\left(t, u_{0}\right)$ be the limit function as in Theorem 1, and $v(t)=v\left(t, v_{0}\right)$ a mild solution to $\left(C P_{T} ; v_{0}\right)$, then

$$
\lim _{\left\|v_{0}-u_{0}\right\| \rightarrow 0}\left\|v\left(t, v_{0}\right)-u\left(t, u_{0}\right)\right\|=0 \text { uniformly on }[0, T) \text {. }
$$

In particular, if $v_{0}=u_{0}$ then $v(t) \equiv u(t)$.

Proof. Putting $s=0$ in (8),

$$
\|v(t)-u(t)\| \leq\left\|v_{0}-u_{0}\right\|+\int_{0}^{t} L(\|v(\tau)-u(\tau)\|) d \tau .
$$

Putting $f(t)=\|v(t)-u(t)\|$ and $F(t)=\left\|v_{0}-u_{0}\right\|+\int_{0}^{t} L(\|v(\tau)-u(\tau)\|) d \tau$, (10) is expressed as

$$
f(t) \leq F(t) .
$$

Thus $F^{\prime}(t)=L(f(t)) \leq L(F(t))$. Hence,

$$
\int_{\left\|v_{0}-u_{0}\right\|}^{F(t)} \frac{d s}{L(s)}=\int_{0}^{t} \frac{F^{\prime}(\tau)}{L(F(\tau))} d \tau \leq \int_{0}^{t} d \tau=t .
$$

Since $\int_{0}^{1} d s / L(s)=\infty, \lim _{\left\|v_{0}-u_{0}\right\| \rightarrow 0} F(t)=0$ uniformly on $[0, T)$. So (9) is obtained. 
Lemma 3.2. Let $u_{0} \in \overline{D(A)}$. Then there exists a unique mild solution to $\left(C P_{T} ; u_{0}\right)$.

Proof. Since $u_{0} \in \overline{D(A)}$, there exists a sequence $\left\{x_{n}\right\} \subset D(A)$ which goes to $u_{0}$. Let $u\left(t, x_{n}\right)$ be the limit function with initial value $x_{n}$. By (9) we have

$$
\lim _{n, m \rightarrow \infty}\left\|u\left(t, x_{n}\right)-u\left(t, x_{m}\right)\right\|=0 \quad \text { uniformly on }[0, T) \text {. }
$$

Putting $u(t)=\lim _{n \rightarrow \infty} u\left(t, x_{n}\right)$, it is clear that $u(t)$ is a mild solution to $\left(C P_{T} ; u_{0}\right)$.

Next, letting $v(t)$ be any mild solution to $\left(C P_{T} ; u_{0}\right)$, using (9) again,

$$
\lim _{n \rightarrow \infty}\left\|v(t)-u\left(t, x_{n}\right)\right\|=0 \text {. }
$$

Thus

$$
\|v(t)-u(t)\| \leq\left\|v(t)-u\left(t, x_{n}\right)\right\|+\left\|u\left(t, x_{n}\right)-u(t)\right\| \rightarrow 0 \quad \text { as } n \rightarrow \infty .
$$

Hence, $v(t) \equiv u(t)$.

From Lemma 3.2, it is easily seen that there exists a unique mild solution $u(t, x)$ to $\left(C P_{\infty} ; x\right)$ for any $x \in \overline{D(A)}$. Then we have the following theorem.

Theorem 2. Let $A$ be a directed L-dissipative operator satisfying the condition (R1). For each $x \in \overline{D(A)}$, let $u(t, x)$ be the unique mild solution to $\left(C P_{\infty} ; x\right)$. Let $T(t)$ be an operator on $\overline{D(A)}$ such that $T(t) x=u(t, x)$. Then $\{T(t) \mid t \geq 0\}$ has semigroup property.

Proof. It is obvious that $T(t) x=x$ and $T(t+s) x=T(t) T(s) x$ for $t, s \geq 0$ and $x \in \overline{D(A)}$. We show that $u(t, x):[0, \infty) \times \overline{D(A)} \rightarrow \overline{D(A)}$ is continuous. Assume that $\left(t_{n}, x_{n}\right),(t, x) \in[0, \infty) \times \overline{D(A)}$ and $\left(t_{n}, x_{n}\right) \rightarrow(t, x)$ as $n \rightarrow$ $\infty$. Choose a sequence $\left\{x_{n}^{\prime}\right\} \subset D(A)$ which converges to $x$. Then by (1),

$$
\begin{aligned}
\left\|u\left(t_{n}, x_{n}\right)-u(t, x)\right\| \leq & \left\|u\left(t_{n}, x_{n}\right)-u\left(t_{n}, x_{n}^{\prime}\right)\right\|+\left\|u\left(t_{n}, x_{n}^{\prime}\right)-u\left(t_{n}, x_{m}^{\prime}\right)\right\| \\
& +\left\|u\left(t_{n}, x_{m}^{\prime}\right)-u\left(t, x_{m}^{\prime}\right)\right\|+\left\|u\left(t, x_{m}^{\prime}\right)-u(t, x)\right\| \\
\leq & \left\|u\left(t_{n}, x_{n}\right)-u\left(t_{n}, x_{n}^{\prime}\right)\right\|+\left\|u\left(t_{n}, x_{n}^{\prime}\right)-u\left(t_{n}, x_{m}^{\prime}\right)\right\| \\
& +\left|A x_{m}^{\prime}\left\|t_{n}-t \mid+\right\| u\left(t, x_{m}^{\prime}\right)-u(t, x) \| .\right.
\end{aligned}
$$

By (9), for any $\varepsilon>0$ there exists a positive integer $N=N(\varepsilon)$ such that

$$
\begin{aligned}
& \left\|u\left(t_{n}, x_{n}\right)-u\left(t_{n}, x_{n}^{\prime}\right)\right\|<\varepsilon / 3, \\
& \left\|u\left(t_{n}, x_{n}^{\prime}\right)-u\left(t_{n}, x_{m}^{\prime}\right)\right\|<\varepsilon / 3, \\
& \left\|u\left(t, x_{m}^{\prime}\right)-u(t, x)\right\|<\varepsilon / 3
\end{aligned}
$$

for $n, m \geq N$. Thus

$$
\begin{aligned}
\left\|u\left(t_{n}, x_{n}\right)-u(t, x)\right\| & \leq \varepsilon / 3+\varepsilon / 3+\left|A x_{m}^{\prime}\right|\left|t_{n}-t\right|+\varepsilon / 3 \\
& =\varepsilon+\left|A x_{m}^{\prime} \| t_{n}-t\right| .
\end{aligned}
$$

Hence

$$
\varlimsup_{n \rightarrow \infty}\left\|u\left(t_{n}, x_{n}\right)-u(t, x)\right\| \leq \varepsilon
$$


Remark. Let $X$ be a real Banach space. In the case $A$ is a continuous mapping from a subset of $[a, b) \times X \quad(a<b \leq \infty)$ into $X$, Iwamiya has given a result that guarantees existence and uniqueness of solutions under a weaker condition than (ii) in $\S 1$ (see [3] in detail) for the nonautonomous differential equation in $X$

giving $(\tau, z)$ in $[a, b) \times X$.

$$
(C P ; \tau, z)\left\{\begin{array}{l}
(d / d t) u(t)=A(t, u(t)), \\
u(t)=z
\end{array}\right.
$$

\section{ACKNOWLEDGMENT}

The author would like to express his hearty thanks to Professor Y. Kōmura for his invaluable advice and constant encouragement and Professors N. Tanaka and $\mathrm{K}$. Kobayasi for their helpful advice.

\section{REFERENCES}

1. Ph. Benilan, Équations d'évolution dans un espace de Banach quelconque et applications, Thèse, Orsay, 1972.

2. K. Furuya, Analyticity of nonlinear semigroups, Israel J. Math. 68 (1989), 89-101.

3. T. Iwamiya, Global existence of solutions to nonautonomous differential equations in Banach spaces, Hiroshima Math. J. 13 (1983), 65-81.

4. Y. Kōmura, Nonlinear semi-groups in Hilbert space, J. Math. Soc. Japan 19 (1967), 493507.

Department of Mathematics, School of Education, Waseda University, 1-6-1 NishiWASEDa, SHINJUKU-KU, TOKYO 169, JAPAN 